\title{
A Spherical Relativistic Anisotropic Compact Star Model
}

\author{
Prakash Chandra Fulara, Archana Sah \\ Department of Mathematics, M B Govt. P G College, Haldwani, Nainital, India \\ Email:pcfsouk@gmail.com,pcfsouk@gmail.com, mailto:archanasah7@gmail.com, archanasah7@gmail.com
}

How to cite this paper: Fulara, P.C. and Sah, A. (2018) A Spherical Relativistic Anisotropic Compact Star Model. International Journal of Astronomy and Astrophysics, 8, 46-67.

https://doi.org/10.4236/ijaa.2018.81004

Received: December 24, 2017

Accepted: March 12, 2018

Published: March 15, 2018

Copyright $\odot 2018$ by authors and Scientific Research Publishing Inc. This work is licensed under the Creative Commons Attribution International License (CC BY 4.0).

http://creativecommons.org/licenses/by/4.0/ (c) (i) Open Access

\begin{abstract}
We provide solutions to Einsteins field equations for a model of a spherically symmetric anisotropic fluid distribution, relevant to the description of compact stars. The central matter-energy density, radial and tangential pressures, red shift and speed of sound are positive definite and are decreasing monotonically with increasing radial distance from the center of matter distribution of astrophysical object. The causality condition is satisfied for complete fluid distribution. The central value of anisotropy is zero and is increasing monotonically with increasing radial distance from the center of the distribution. The adiabatic index is increasing with increasing radius of spherical fluid distribution. The stability conditions in relativistic compact star are also discussed in our investigation. The solution is representing the realistic objects such as SAXJ1808.4-3658, HerX-1, 4U1538-52, LMC X-4, CenX-3, VelaX-1, PSRJ1614-2230 and PSRJ0348+0432 with suitable conditions.
\end{abstract}

\section{Keywords}

Anisotropic Fluid Distribution, Non Singular Solutions, Einstein's Field Equations, Super Dense Star, General Relativity

\section{Introduction}

The General Theory of Relativity provides the mathematical platform for describing the physical world generated by highly compact astrophysical objects. According to Einstein's relativistic theory, the existence of matter and its gravitational field causes to exist space-time gets curvature. When an incoherent matter like dust is contracted and condensed, a condition is reached where gas degeneracy pressure, thermal pressure (negligible with respect to gas degeneracy pressure) and gravitational pressure are in equilibrium. This equilibrium state forms a dense astrophysical object like neutron star, white dwarf, quark star, strange star etc. In order to understand the structural and physical properties of 
this dense astrophysical object, it is needed to find out the exact solution of Einstein's relativistic field equation. Therefore exploration of anisotropic solution of Einstein's relativistic field equation attracts the researchers to do work in this field. In 1916 Schwarzschild [1] solved Einstein's field equations for describing the internal structure of a dense astrophysical object. He gave two solutions, one corresponding to external space time geometry and other corresponding to internal geometrical structure of fluid distribution by assuming constant matter energy density. But this solution has singularity at the center. Due to non-linearity and complexity of the differential equation, it is difficult to find the exact solution of these equations. Tolman [2] gave eight solutions for describing the internal structure and physical properties of super dense astrophysical object. The first solution corresponds to Einstein's universe of static configuration having constant density and pressure. The second and third solutions correspond to Schwarzschild-de Sitter and Schwarzschild interior solutions respectively. The fifth and sixth solutions gave infinite constant density and pressure while the fourth and seventh solutions are physically acceptable. With passage of time, the number of non-singular solutions of Einstein's field equation for theoretical description of super dense astrophysical objects in static case were made by various researchers.

Buchdahl [3] gave well known limit of the compactness for stable fluid distribution and limit of emission of radiation due to gravitational collapse of the distribution. This limit plays an important role for the stability of fluid distribution. Delgaty-Lake [4] examined 127 solutions and concluded that only 16 solutions were physically accepted against six physical properties namely non-singularity, isotropy in pressure, positive definiteness of central pressure and density, zero pressure at the surface, decreasing pressure and density with increasing radial distance. In addition to these properties, only nine solutions (Tolman, Patwardhan and Vaidya [5], Mehra [6], Kuchowicz [7], Matese and Whitman [8], Durgapals two solutions [9], Nariai [10], Goldman [11]) gave monotonically decreasing speed of sound with increasing radial distance. Other solutions (Adler [12], Heintzmann [13], Finch and Skea [14] etc.) are not well behaved under these examining properties. Maurya and Gupta [15], Pant [16], Ivanov [17] [18], Pant et al. [19] gave physically accepted isotropic solutions of Einstein's non-linear differential equations of relativistic field theory.

The radial pressure of fluid distribution may be different from the tangential pressure which may lead anisotropy in pressure. In the study of the properties of internal structure of a super dense astrophysical object, the local anisotropy is commonly used by the researchers in their solutions. The phase transition of fluid, existence of a solid core and other physical phenomena create local anisotropy in the pressure of the fluid. The surface red-shift of an astrophysical object, its stable mass and other physical properties may be affected by by anisotropy present in fluid distribution within that object. Combination of effect of anisotropy with equation of hydrostatic equilibrium investigates role of equations of state for fluid distribution. 
Some researchers, Sah and Chandra [20], Komathiraj and Maharaj [21], Herrera et al. [22], Thirukkanesh and Regel [23], Chaisi and Maharaj [24], Sunzu et al. [25], Maurya and Gupta [26] and Bhar et al. [27] etc. gave physically accepted solutions of Einstein's field equations for anisotropic fluid distribution. The researchers Sah and Chandra [28], Whitman and Burch [29], Tikekar [30], Ivanov [31], Gupta and Kumar [32], Herrera et al. [33] [34] [35], Tewari and Charan [36] [37] [38], Tewari [39], Ivanov [40] [41] [42] [43], Maurya and Gupta [44] etc gave remarkable work in relativistic astrophysics. The stellar model given in this paper is the contribution of the work related to charged fluid distribution, done by Sah and Chandra. In this work, we discuss physically accepted solutions for anisotropic fluid distribution in addition with energy conditions, variation of mass with central density, equation of state and mass-radius relationship in contest of some astrophysical objects, SAXJ1808.4-3658, HerX-1, 4U1538-52 and LMC X-4.

The whole work of this paper is divided into nine sections. The second section is comprised of Einstein's non-linear differential equations of field theory. Besides this the physical parameters like matter-energy density, radial and tangential pressures, anisotropy, surface and central red shifts are expressed in this section. In third section the parameters of physically acceptable non-singular solutions are given. A non-singular physically acceptable solution of Einstein's field equations given by Sah and Chandra [28] for anisotropic fluid distribution is incooperated in fourth section. The physical properties of the solution are given in fifth section. The matching of interior metric of the distribution with the exterior metric given by Schwarzschild is included in sixth section. In seventh section the tabular and graphical representation of physical parameters of our model of super dense star corresponding non-singular and physically accepted solutions are presented. The stability criteria are given in eighth section. In ninth section, some conclusions of whole work presented in this paper are given.

\section{Einstein's Field Equation of General Relativity}

The relationship between gravity due to existing fluid material and geometry of space-time, contained in the Einstein's non-linear differential equations for relativistic field theory is given by

$$
\mathscr{S}_{i j}-\frac{1}{2} g_{i j}=-\frac{8 \pi \mathscr{f}}{c^{4}} \cdot \mathscr{T}_{i j}
$$

where $\mathscr{T}_{i j}$, the energy momentum tensor for spherically anisotropic fluid distribution is defined as

$$
\mathscr{T}_{i j}=\left(\rho c^{2}+p_{r}\right) u_{i} u_{j}-p_{t} g_{i j}+\left(p_{r}-p_{t}\right) x_{i} x_{j}
$$

where $\rho$ is the proper density, $p_{r}$ and $p_{t}$ are radial and tangential pressures of an anisotropic fluid along and perpendicular to $u_{\mu}$ (time-like four-velocity vector) respectively, $x_{\mu}$ is the unit space like vector along radial vector and $g_{v}^{\mu}$ metric tensor such that 


$$
g_{i j} u^{i} u^{j}=1
$$

For spherically symmetric stable fluid distribution, the metric element can be expressed as

$$
\mathrm{d} s^{2}=-\mathscr{\beta}^{2} \mathrm{~d} r^{2}-r^{2}\left(\mathrm{~d} \theta^{2}+\sin ^{2} \theta \mathrm{d} \phi^{2}\right)+\mathscr{C}^{2} \mathrm{~d} t^{2}
$$

where $\mathscr{A}$ and $\mathscr{B}$ are metric coefficients depending on radial distance $r$ only.

Incorporating Equation (2) and Equation (3) with Equation (1), we get the following equations

$$
\begin{aligned}
& \frac{8 \pi \mathscr{Z}}{c^{4}} p_{r}=\frac{1}{\mathscr{R}^{2}}\left(\frac{2 \mathscr{C}^{\prime}}{r \cdot /}+\frac{1}{r^{2}}\right)-\frac{1}{r^{2}}
\end{aligned}
$$

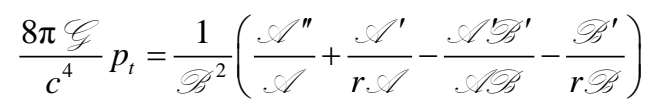

$$
\begin{aligned}
& \frac{8 \pi \mathscr{f}}{c^{2}} \rho=-\frac{1}{\mathscr{\beta}^{2}}\left(\frac{2 \beta^{\prime}}{r}-\frac{1}{r^{2}}\right)+\frac{1}{r^{2}} \\
& \frac{8 \pi \mathscr{f}}{c^{4}}\left(p_{t}-p_{r}\right)=\Delta(r)=\frac{\delta(r)}{\mathscr{\beta}^{2}}
\end{aligned}
$$

where

$$
\delta(r)=\left(\frac{\mathscr{C}^{\prime \prime}}{\mathscr{A}}-\frac{\mathscr{B}^{\prime}}{r \mathscr{B}}-\frac{\mathscr{\mathscr { O }} \mathscr{P}^{\prime}}{\mathscr{\mathscr { B }}}-\frac{\mathscr{C}^{\prime}}{r \mathscr{C}}-\frac{1}{r^{2}}\right)+\frac{\mathscr{B}^{2}}{r^{2}}
$$

In the above equations, (') denotes the derivative against radial distance $r$.

The redshift due to gravity of dense spherically symmetric fluid distribution is given by

$$
1+\not z=g_{00}^{\frac{-1}{2}}
$$

which turns out the gravitational redshift $(\not{F})$ for compact stellar object and it's surface redshift $\left(z_{\Sigma}\right)$ as

$$
\mathscr{C}=\frac{c}{\mathscr{A}}-1
$$

and

$$
F_{\Sigma}=\frac{1}{\left(1-2 \rho_{p}\right)^{\frac{1}{2}}}-1
$$

where Schwarzchild parameter or compactness, $\mathscr{S}_{p}=\frac{C_{\mathscr{C}} \mathbb{}}{c^{2} r_{\Sigma}}, r_{\Sigma}$ is the radius of spherically symmetric stellar fluid configuration of mass . $/$.

\section{Physical Acceptability Conditions for Stable Stellar Configuration}

The non-singular solution of Einstein's field equation must satisfy the following physically accepted conditions

1) There is no singularity in the solution interior and on the surface of the 
astrophysical object. For these metric coefficients. $\mathscr{A}$ and $\mathscr{B}$, central pressure and central density should be positive definite throughout the interior of the object i.e. $\mathscr{C}, \mathscr{P} \geq 0, \rho_{0}>0$ and $p_{0}>0$.

2) The density, radial and tangential pressures should be maximum at the center and decreased monotonically on moving from center to the surface of the fluid object i.e.

a) at center $\left(\frac{\mathrm{d} p_{r}}{\mathrm{~d} r}\right)_{0}=0=\left(\frac{\mathrm{d} p_{t}}{\mathrm{~d} r}\right)_{0}=\left(\frac{\mathrm{d} \rho}{\mathrm{d} r}\right)_{0}$ and $\left(\frac{\mathrm{d}^{2} p_{r}}{\mathrm{~d} r^{2}}\right)_{0},\left(\frac{\mathrm{d}^{2} p_{t}}{\mathrm{~d} r^{2}}\right)_{0}$ and $\left(\frac{\mathrm{d}^{2} \rho}{\mathrm{d} r^{2}}\right)_{0}<0$ for maximum value of $\rho, p_{r}$ and $p_{t}$. such that the radial pressure gradient, $\frac{\mathrm{d} p_{r}}{\mathrm{~d} r}$ is negative for $0 \leq r \leq r_{\Sigma}$.

b) For monotonically decreasing density and pressures $\left(\frac{\mathrm{d} p_{r}}{\mathrm{~d} r}\right)_{0},\left(\frac{\mathrm{d} p_{t}}{\mathrm{~d} r}\right)_{0},\left(\frac{\mathrm{d} \rho}{\mathrm{d} r}\right)_{0} \leq 0$ for $0 \leq \frac{r}{r_{\Sigma}} \leq 1$.

3) At boundary radial pressure, $p_{r}$ shold be equal to zero while tangential pressure, $p_{t}$ may not be equal to zero i.e. pressure anisotropy is zero at the center i.e. $\Delta_{0}=0$. The pressure anisotropy $\Delta$ should be increased with radial distance from the center of fluid distribution and at the surface it must be $\Delta_{\Sigma}=\left(p_{t}\right)_{\Sigma}$ for zero surface radial pressure.

4) The null energy condition (NEC) $\rho \geq 0$, weak energy condition (WEC) $0 \leq \frac{p_{r}}{\rho c^{2}} \leq 1, \quad 0 \leq \frac{p_{t}}{\rho c^{2}} \leq 1$ and strong energy condition (SEC) $0 \leq \frac{p_{r}}{\rho c^{2}}+2 \frac{p_{t}}{\rho c^{2}} \leq 1$ with $\frac{p_{r}}{\rho c^{2}}>0$ and $\frac{p_{t}}{\rho c^{2}}>0$ should be satisfied throughout within the fluid object.

5) The causality conditions $0 \leq \sqrt{\frac{\mathrm{d} p_{r}}{c^{2} \mathrm{~d} \rho}}<1$ and $0 \leq \sqrt{\frac{\mathrm{d} p_{t}}{c^{2} \mathrm{~d} \rho}}<1$ must be satisfied throughout the stellar object. The speed of sound must be decreased monotonically on increasing the radial distance and increased with increasing density i.e. $\frac{\mathrm{d}}{\mathrm{d} r}\left(\frac{\mathrm{d} p_{r}}{\mathrm{~d} \rho}\right)<0$ or $\left(\frac{\mathrm{d}^{2} p_{r}}{\mathrm{~d} \rho^{2}}\right)>0$ and $\frac{\mathrm{d}}{\mathrm{d} r}\left(\frac{\mathrm{d} p_{t}}{\mathrm{~d} \rho}\right)<0$ or $\left(\frac{\mathrm{d}^{2} p_{t}}{\mathrm{~d} \rho^{2}}\right)>0$.

Thus the equation of state for highly dense astrophysical object indicates that the speed of sound should be decreased with increasing radial distance.

6) The realistic adiabatic index $\Gamma_{r}=\frac{\rho c^{2}+p_{r}}{p_{r}} \frac{\mathrm{d} p_{r}}{c^{2} \mathrm{~d} \rho}$ and $\Gamma_{t}=\frac{\rho c^{2}+p_{t}}{p_{t}} \frac{\mathrm{d} p_{t}}{c^{2} \mathrm{~d} \rho}$ should be positive and radial adiabatic index, $\Gamma_{r}$ should be greater than $\frac{4}{3}$.

7) The gravitational red shift must have positive finite value and be decreased monotonically with increasing the radial distance.

8) The difference of the square of the radial speed of sound and tangential speed of sound $\frac{v_{t}^{2}}{c^{2}}-\frac{v_{r}^{2}}{c^{2}}$ should lie between -1 and 0 for the matter within the object. 
The method of solving the Einstein equations is to choose ansatze for the two metric functions. Then the three Einstein Equations (4)-(6) give expressions for the matter components $\rho, p_{r}$ and $p_{t}$. The two metric components are so related to allow simple forms for the physical variables. There is no integration in fact.

\section{A Physically Acceptable Non-Singular Solution}

The non-singular solution of Einstein's field Equations (4) to (7) presented by Sah and Chandra [28] is given by

$$
\begin{aligned}
& \mathscr{\gamma}=\beta+\gamma\left(1-\alpha r^{2}\right)^{-1} \\
& =\left(1-\alpha r^{2}\right)^{-2}
\end{aligned}
$$

Here $\alpha$ has dimension (length) $)^{-2}$ and $\beta, \gamma$ are dimensionless constants.

Using Equation (12) and Equation (13), Equation (4) gives radial pressure of the fluid inside the object as

$$
\frac{8 \pi \mathscr{g}}{c^{4}} p_{r}=\alpha\left\{\frac{4 \gamma\left(1-\alpha r^{2}\right)^{3}}{\beta\left(1-\alpha r^{2}\right)+\gamma}+\alpha^{3} r^{6}-4 \alpha^{2} r^{4}+6 \alpha r^{2}-4\right\}
$$

Equation (12) and Equation (13) with Equation (5) give tangential pressure as

$$
\frac{8 \pi \mathscr{f}}{c^{4}} p_{t}=4 \alpha\left\{\frac{\gamma\left(1-\alpha r^{2}\right)^{3}}{\beta\left(1-\alpha r^{2}\right)+\gamma}-\left(1-\alpha r^{2}\right)^{3}\right\}
$$

Equation (6) gives the matter energy density for our solution as

$$
\frac{8 \pi}{c^{2}} \rho=\alpha\left(12-30 \alpha r^{2}+28 \alpha^{2} r^{4}-9 \alpha^{3} r^{6}\right)
$$

The red shift and anisotropy of astrophysical fluid distribution are given by

$$
\begin{gathered}
z=\frac{(c-\beta)\left(1-\alpha r^{2}\right)-\gamma}{\beta\left(1-\alpha r^{2}\right)+\gamma} \\
\Delta=\alpha\left(6 \alpha r^{2}-8 \alpha^{2} r^{4}+3 \alpha^{3} r^{6}\right)
\end{gathered}
$$

The radial derivatives of pressures $p_{r}^{\prime}$ and $p_{t}^{\prime}$ given by Equations (14) and (15) are

$$
\begin{gathered}
\frac{8 \pi \mathscr{f}}{c^{4}} p_{r}^{\prime}=-\alpha^{2} r\left[\frac{8 \gamma\left(1-\alpha r^{2}\right)^{2}\left\{2 \beta\left(1-\alpha r^{2}\right)+3 \gamma\right\}}{\left\{\beta\left(1-\alpha r^{2}\right)+\gamma\right\}^{2}}-12+16 \alpha r^{2}-6 \alpha^{2} r^{4}\right] \\
\frac{8 \pi f}{c^{4}} p_{t}^{\prime}=-\alpha^{2} r\left[\frac{\gamma\left(1-\alpha r^{2}\right)^{2}\left\{2 \beta\left(1-\alpha r^{2}\right)+3 \gamma\right\}}{\left\{\beta\left(1-\alpha r^{2}\right)+\gamma\right\}^{2}}-3\left(1-\alpha r^{2}\right)^{2}\right]
\end{gathered}
$$

The radial derivative of matter energy density given by Equation (16) is

$$
\frac{8 \pi \mathscr{f}}{c^{2}} \rho^{\prime}=-\alpha^{2} r\left(60-112 \alpha r^{2}+54 \alpha^{2} r^{4}\right)
$$


The radial derivatives of redshift and anisotropy given by Equation (17) and Equation (18) respectively are

$$
\begin{gathered}
z^{\prime}=\frac{-2 c \alpha \gamma r}{\left\{\beta\left(1-\alpha r^{2}\right)+\gamma\right\}^{2}} \\
\Delta^{\prime}=2 \alpha^{2} r\left(6-16 \alpha r^{2}+9 \alpha^{2} r^{4}\right)
\end{gathered}
$$

\section{Conditions for Model Parameters for Physical Acceptable Solution}

For positive value of metric coefficient $\mathscr{A}$ i.e. $/ \mathscr{C}_{0}>0$ is possible if $(\beta+\gamma)>0$. The monotonically increasing nature of metric coefficients and $\mathscr{P}$ with increasing radial distance $r$ for suitable choice of constants $\alpha, \beta$, and $\gamma$ are shown in Figure 1(a) and Figure 1(b). The values of pressure $p$, density $\rho$ and red shift $\%$ at the center are given by

$$
\begin{gathered}
\frac{8 \pi \mathscr{f}}{c^{4}}\left(p_{r}\right)_{0}=\frac{-4 \alpha \beta}{\beta+\gamma}=\frac{8 \pi \mathscr{f}}{c^{4}}\left(p_{t}\right)_{0} \\
\frac{8 \pi \mathscr{f}}{c^{2}} \rho_{0}=12 \alpha \\
z_{0}=\frac{c-\beta-\gamma}{\beta+\gamma}
\end{gathered}
$$

For positive values of pressure $p_{0}$, density $\rho_{0}$ and redshift $\%_{0}, \alpha>0$, $\beta+\gamma<c$ and $\beta<0$.

From Equations (19) to (23), the radial derivatives of pressure, density, red shift and anisotropy at the center of fluid distribution are zero.

From Equation (19) and Equation (20), at the center of fluid distribution

$$
\frac{8 \pi \mathscr{f}}{c^{4}}\left(p_{r}^{\prime \prime}\right)_{0}=-4 \alpha^{2} \frac{\left(3 \gamma^{2}-4 \beta \gamma-3 \beta^{2}\right)}{(\beta+\gamma)^{2}}
$$

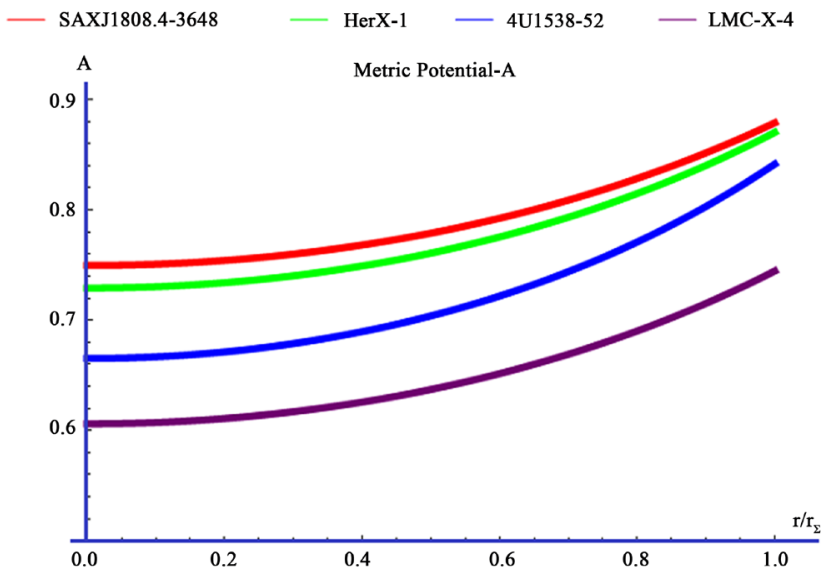

(a)

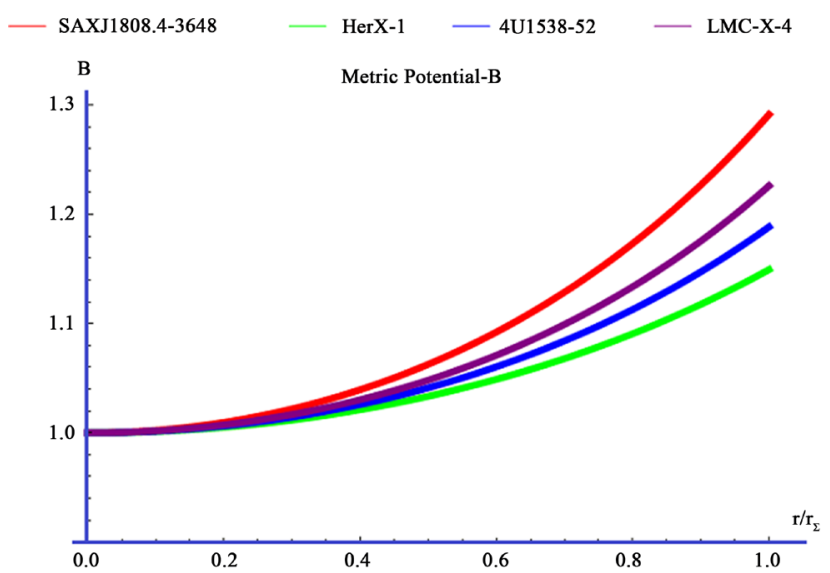

(b)

Figure 1. (a): The variation of Metric Potential “. "; (b): The variation of Metric Potential “ $\mathscr{\not}$ ” against $\frac{r}{r_{\Sigma}}$. 


$$
\frac{8 \pi \mathscr{G}}{c^{4}}\left(p_{t}^{\prime \prime}\right)_{0}=8 \alpha^{2} \beta \frac{(3 \beta+4 \gamma)}{(\beta+\gamma)^{2}}
$$

For maximum values of radial and tangential pressures at the center, the conditions of model parameters are $\left(p_{r}^{\prime \prime}\right)_{0}<0$ i.e. $\frac{-2-\sqrt{13}}{3}<\frac{\beta}{\gamma}<\frac{-2+\sqrt{13}}{3}$ and $\left(p_{t}^{\prime \prime}\right)_{0}<0$ i.e. $-\frac{4}{3}<\frac{\beta}{\gamma}$.

From Equation (21)

$$
\frac{8 \pi \mathscr{f}}{c^{4}}\left(\rho^{\prime \prime}\right)_{0}=-60 \alpha^{2}
$$

which shows that for all values of parameters, the matter energy density is maximum at the center of the fluid distribution i.e. $\left(\rho^{\prime \prime}\right)_{0}<0$.

At the center, the equations of state for matter distribution are given by

$$
\begin{aligned}
& \left(\frac{p_{r}}{c^{2} \rho}\right)_{0}=-\frac{\beta}{3(\beta+\gamma)} \\
& \left(\frac{p_{t}}{c^{2} \rho}\right)_{0}=-\frac{\beta}{3(\beta+\gamma)}
\end{aligned}
$$

The central equation of state should obey the conditions $0<\left(\frac{p_{r}}{c^{2} \rho}\right)_{0} \leq 1$ and $0<\left(\frac{p_{t}}{c^{2} \rho}\right)_{0} \leq 1$ which gives $-\frac{4}{3} \leq \frac{\beta}{\gamma}$.

From Equation (19) and Equation (21)

$$
\left(\frac{\mathrm{d} p_{r}}{c^{2} \mathrm{~d} \rho}\right)_{0}=\frac{\left(3 \gamma^{2}-2 \beta \gamma-3 \beta^{2}\right)}{15(\beta+\gamma)^{2}}
$$

and from Equation (20) and Equation (22)

$$
\left(\frac{\mathrm{d} p_{t}}{c^{2} \mathrm{~d} \rho}\right)_{0}=-\frac{\left(4 \gamma^{2}-25 \beta \gamma-20 \beta^{2}\right)}{60(\beta+\gamma)^{2}}
$$

The causality conditions $0<\left(\frac{\mathrm{d} p_{r}}{c^{2} \mathrm{~d} \rho}\right)_{0} \leq 1$ and $0<\left(\frac{\mathrm{d} p_{t}}{c^{2} \mathrm{~d} \rho}\right)_{0} \leq 1$ are satisfied at the center which gives the condition for model parameters $-\frac{6}{7}<\frac{\beta}{\gamma}<-\frac{10}{9}$.

From Equation (22) and Equation (23)

$$
\begin{gathered}
\left(z^{\prime \prime}\right)_{0}=-\frac{2 \alpha c \gamma}{(\beta+\gamma)^{2}} \\
\left(\Delta^{\prime \prime}\right)_{0}=12 \alpha^{2}
\end{gathered}
$$

For maximum value of red shift at the center, $\left(z^{\prime \prime}\right)_{0}<0$ i.e. $\gamma>0$. Since $\left(\Delta^{\prime \prime}\right)_{0}>0$, the anisotropy is minimum at the center for all values of model parameters.

It is observed that for model parameters given by 


$$
\alpha>0, \beta<0, \gamma>0, c>\beta+\gamma>0,-\frac{4}{3}<\frac{\beta}{\gamma}<\frac{-10}{3} \text { and }-\frac{6}{7}<\frac{\beta}{\gamma}<0 \text {, }
$$

the metric coefficients $\mathscr{C}$ and $\mathscr{P}$ increase monotonically with increasing radial distance $r$ as illustrated by (Figure 1(a) and Figure 1(b)). The matter energy density $\rho$ reduces monotonically with increasing radial distance $r$ as shown in the (Figure 2) while radial and pressures decreases monotonically with increasing radial distance $r$ such that the radial pressure becomes zero at the surface of the fluid distribution (Figure 3(a) and Figure 3(b)). The pressure density ratios are always positive and less than one throughout the distribution (Figure 4(a) and Figure 4(b)). The red shift and anisotropy with zero central value increases with increasing radial distance $r$ (Figure 5, Figure 6).

\section{Model Parameters by Matching Conditions}

For the stable astrophysical fluid distribution the interior metric must be matched with the Schwarzschild exterior metric given by

$$
\mathrm{d} s^{2}=\left(1-\frac{2}{c^{2} r}\right) c^{2} \mathrm{~d} t^{2}-\left(1-\frac{2}{c^{2} r}\right)^{-1} \mathrm{~d} r^{2}-r^{2}\left(\mathrm{~d} \theta^{2}+\sin ^{2} \theta \mathrm{d} \phi^{2}\right)
$$

For this the metric coefficients must be continuous (First fundamental form) and differentiable (Second fundamental form) at the surface at $r=r_{\Sigma}$ and $\left(p_{r}\right)_{r_{\Sigma}}=0$.

Thus

$$
\begin{aligned}
& \alpha=\frac{1}{r_{\Sigma}^{2}}\left(1-\Psi^{\frac{1}{2}}\right) ; 0<X<1 \\
& \beta=-\frac{C\left(1+\Psi^{\frac{1}{2}}+\Psi-3 \Psi^{\frac{3}{2}}\right)}{4 \Psi}
\end{aligned}
$$

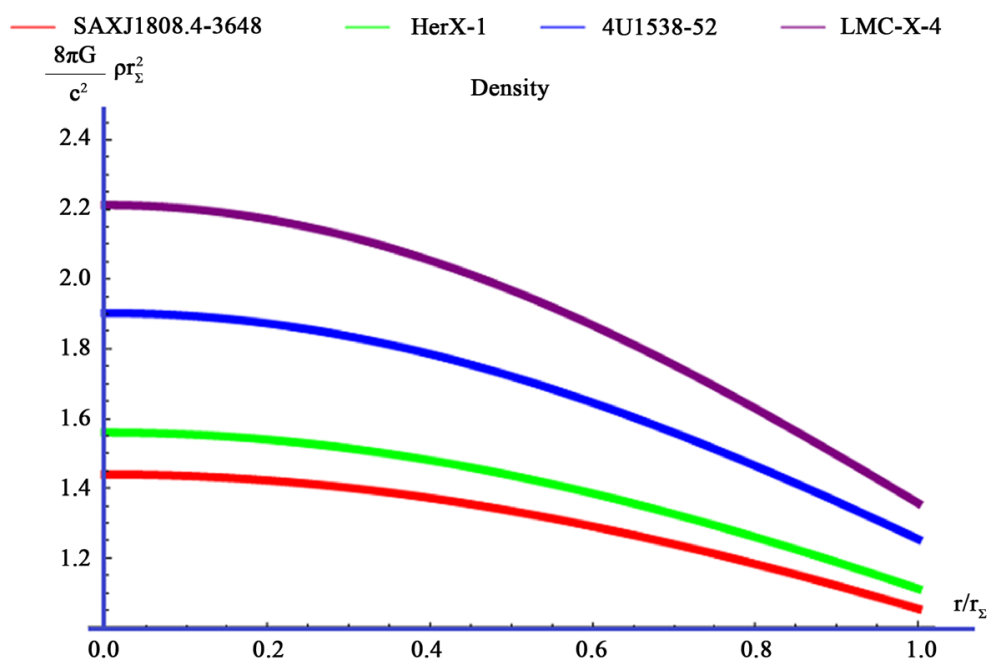

Figure 2. Variation of Density “ $\rho$ ” against $\frac{r}{r_{\Sigma}}$. 


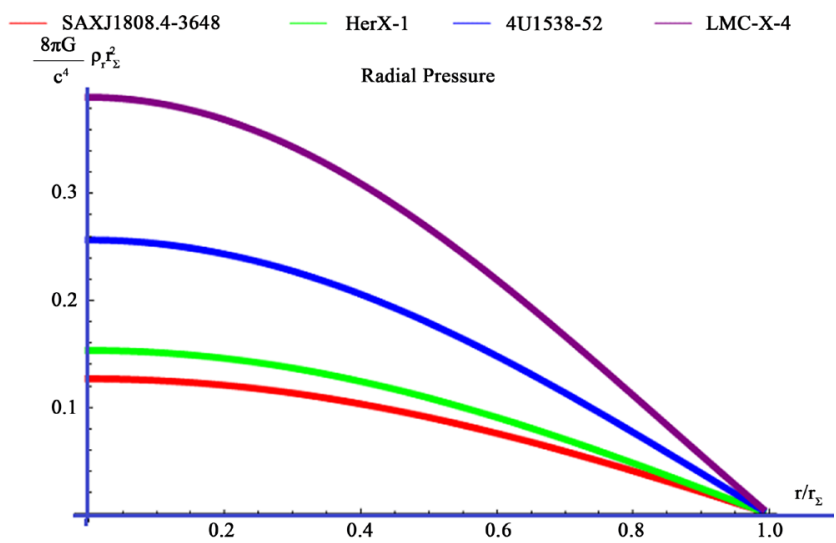

(a)

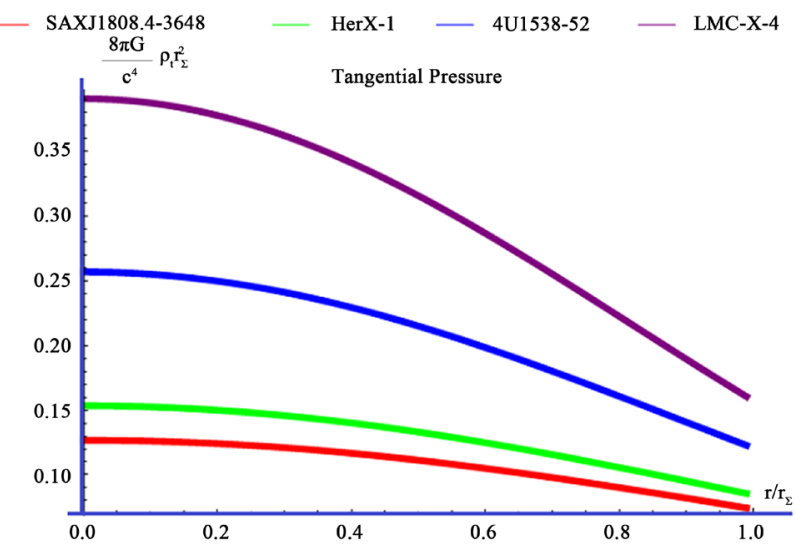

(b)

Figure 3. (a): Variation of Radial Pressure $p_{r}$ against $\frac{r}{r_{\Sigma}} ;(\mathrm{b})$ : Variation of tangential Pressure $p_{t}$ with $\frac{r}{r_{\Sigma}}$.

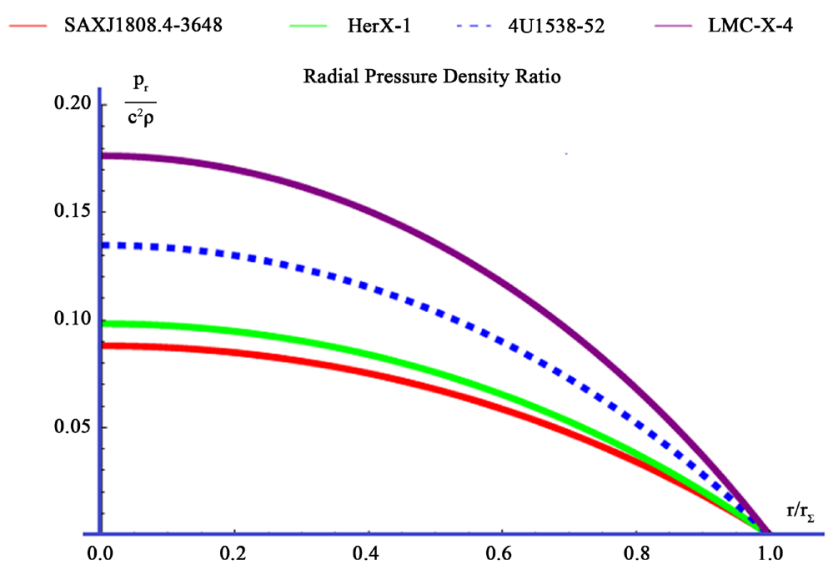

(a)

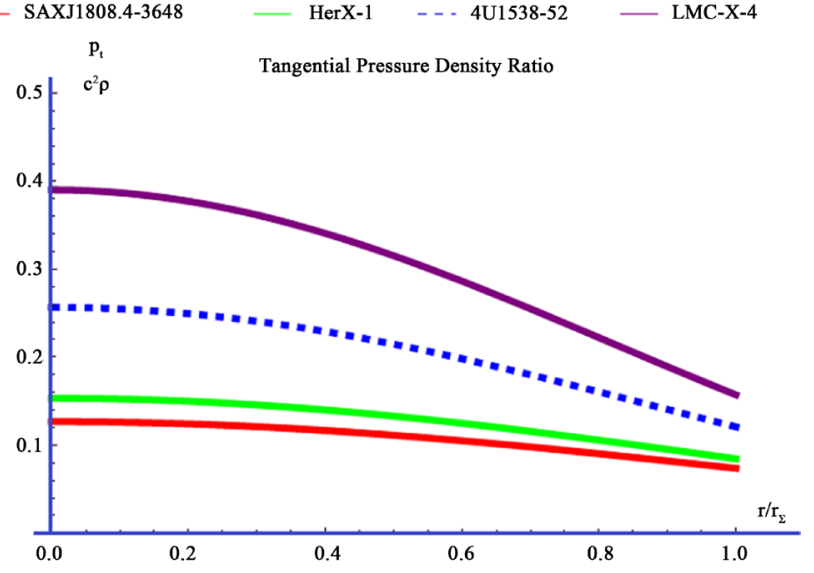

(b)

Figure 4. (a):Variation of $\frac{p_{r}}{c^{2} \rho}$ with $\frac{r}{r_{\Sigma}} ;(\mathrm{b})$ : Variation of $\frac{p_{t}}{c^{2} \rho}$ with $\frac{r}{r_{\Sigma}}$.

$$
\frac{\beta}{\gamma}=-\frac{1+\Psi^{\frac{1}{2}}+\Psi-3 \Psi^{\frac{3}{2}}}{\Psi^{\frac{1}{2}}\left(1+\Psi^{\frac{1}{2}}+\Psi+\Psi^{\frac{3}{2}}\right)}
$$

where $\Psi=1-2 \mathscr{S}_{p} ; \mathscr{S}_{p}=\frac{\mathscr{C} / l}{c^{2} r_{\Sigma}}$, Schwarzchild parameter. Equation (38) to Equation (41) represent the model parameters obtaining from these boundary conditions.

\section{Tabular and Graphical Representation of Physical Quantities}

From Equation (38) to Equation (41) the model parameters $\Psi, \beta, \gamma$ and $\frac{\beta}{\gamma}$ with $\alpha r_{\Sigma}^{2}=0.12$ or $\mathscr{S}_{p}=0.1133$ for an astrophysical object 


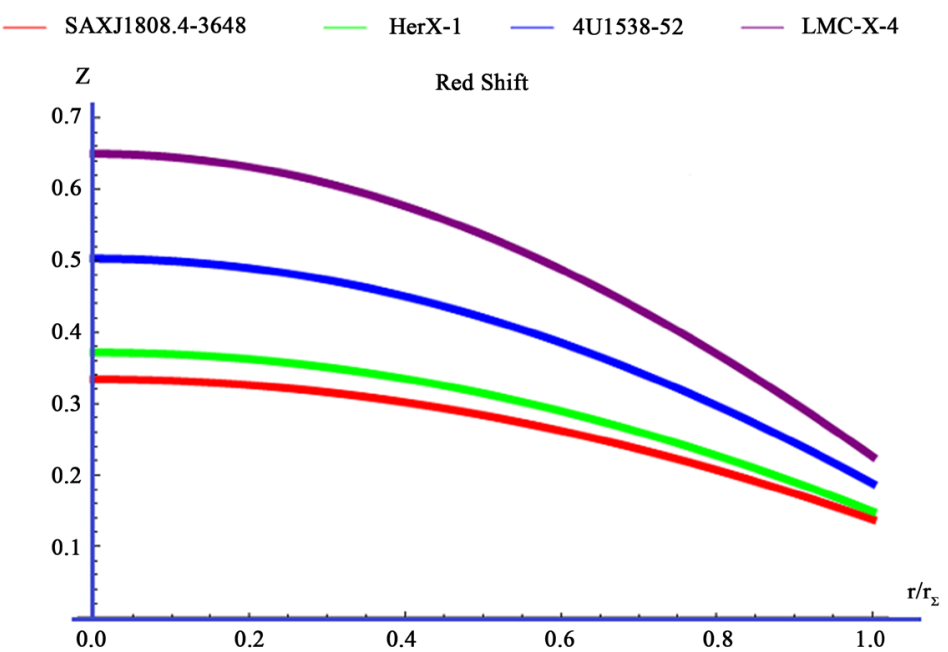

Figure 5. Variation of Red Shift with $\frac{r}{r_{\Sigma}}$.

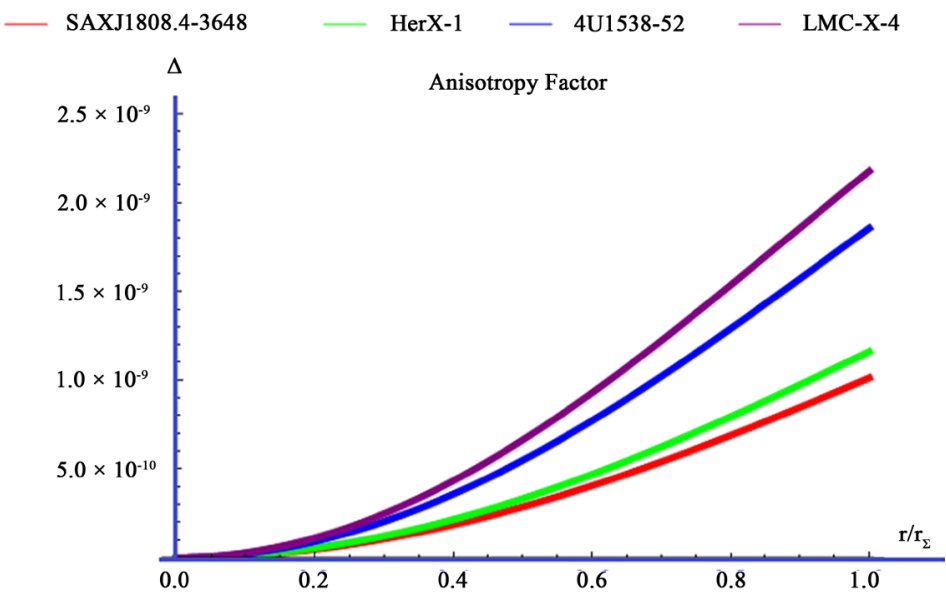

Figure 6. Variation of Anisotropy factor with $\frac{r}{r_{\Sigma}}$.

SAXJ1808.4-3658 are $0.7734,-0.1979 c, 0.9475 c$ and -0.2089 respectively. The physical quantities estimated in our model for astrophysical objects SAXJ1808.4-3658, HerX-1, 4U1538-52 and LMC X-4 are illustrated by means of Table 1, Table 2 and Figures 1(a)-13(b) corresponding to respective model parameters given in Table 3.

With the help of model parameters for astrophysical objects SAXJ1808.4-3658, HerX-1, 4U1538-52, LMC-X-4, CenX-3, VelaX-1, PSRJ1614-2230 and PSRJ0348+0432 given in Table 3 with compactness $\mathscr{T}_{p}$, a super dense compact star model can be made which may be useful for further study of the different properties of various astrophysical objects.

\section{Stability Criterion}

In this section our aim is to determine the physical requirement of realistic solution featuring the stability of compact stellar or astrophysical objects. 
Table 1. Variation of $\frac{\mathscr{C}}{c}, \mathscr{\beta}, \rho, p_{r}, p_{t}, \not{C}$ and $\Delta$ with $\frac{r}{r_{\Sigma}}$ for an astrophysical object SAXJ1808.4-3658 corresponding to parameters $\alpha r_{\Sigma}^{2}=0.12$ or $\mathscr{S}_{p}=0.1133$.

\begin{tabular}{ccccccccc}
\hline S.N. & $\frac{r}{r_{\Sigma}}$ & $\frac{\mathscr{I}}{c}$ & $\mathscr{B}$ & $\frac{8 \pi \mathscr{C}}{c^{2}} \rho r_{\Sigma}^{2}$ & $\frac{8 \pi \mathscr{C}}{c^{4}} p_{r} r_{\Sigma}^{2}$ & $\frac{8 \pi \mathscr{C}}{c^{4}} p_{t} r_{\Sigma}^{2}$ & \multirow{Z}{*}{} & $\Delta$ \\
\hline 1 & 0.0 & 0.6061 & 1.0000 & 1.4400 & 0.1267 & 0.1267 & 0.3340 & 0.0000 \\
2 & 0.1 & 0.6072 & 1.0024 & 1.4357 & 0.1252 & 0.1261 & 0.3320 & $1.2000 \times 10^{-11}$ \\
3 & 0.2 & 0.6105 & 1.0096 & 1.4228 & 0.1207 & 0.1241 & 0.3259 & $4.7502 \times 10^{-11}$ \\
4 & 0.3 & 0.6162 & 1.0219 & 1.4015 & 0.1133 & 0.1210 & 0.3158 & $1.0602 \times 10^{-10}$ \\
5 & 0.4 & 0.6242 & 1.0395 & 1.3721 & 0.1032 & 0.1167 & 0.3018 & $1.8637 \times 10^{-10}$ \\
6 & 0.5 & 0.6347 & 1.0628 & 1.3350 & 0.0905 & 0.1113 & 0.2838 & $2.8698 \times 10^{-10}$ \\
7 & 0.6 & 0.6479 & 1.0923 & 1.2906 & 0.0756 & 0.1050 & 0.2620 & $4.0589 \times 10^{-10}$ \\
8 & 0.7 & 0.6640 & 1.1288 & 1.2397 & 0.0588 & 0.0979 & 0.2364 & $5.4074 \times 10^{-10}$ \\
9 & 0.8 & 0.6832 & 1.1733 & 1.1828 & 0.0404 & 0.0902 & 0.2071 & $6.8885 \times 10^{-10}$ \\
10 & 0.9 & 0.7058 & 1.2269 & 1.1208 & 0.0208 & 0.0821 & 0.1742 & $8.4721 \times 10^{-10}$ \\
11 & 1.0 & 0.7324 & 1.2913 & 1.0545 & 0.0000 & 0.0736 & 0.1379 & $1.0125 \times 10^{-9}$ \\
\hline
\end{tabular}

Table 2. $\frac{p_{r}}{c^{2} \rho}, \frac{p_{t}}{c^{2} \rho}, \frac{\mathrm{d} p_{r}}{c^{2} \mathrm{~d} \rho}, \frac{\mathrm{d} p_{t}}{c^{2} \mathrm{~d} \rho}, \frac{\left(\rho c^{2}+p_{r}\right)}{p_{r}} \frac{\mathrm{d} p_{r}}{c^{2} \mathrm{~d} \rho}, \frac{\left(\rho c^{2}+p_{t}\right)}{p_{t}} \frac{\mathrm{d} p_{t}}{c^{2} \mathrm{~d} \rho} \quad$ and $\frac{v_{t}^{2}}{c^{2}}-\frac{v_{r}^{2}}{c^{2}}$ for an astrophysical object SAXJ1808.4-3658 corresponding to parameters $\alpha r_{\Sigma}^{2}=0.12$ or $\mathscr{T}_{p}=0.1133$.

\begin{tabular}{ccccccccc}
\hline S.N. & $\frac{r}{r_{\Sigma}}$ & $\frac{p_{r}}{c^{2} \rho}$ & $\frac{p_{t}}{c^{2} \rho}$ & $\frac{\mathrm{d} p_{r}}{c^{2} \mathrm{~d} \rho}$ & $\frac{\mathrm{d} p_{t}}{c^{2} \mathrm{~d} \rho}$ & $\frac{\left(\rho c^{2}+p_{r}\right)}{p_{r}} \frac{\mathrm{d} p_{r}}{c^{2} \mathrm{~d} \rho}$ & $\frac{\left(\rho c^{2}+p_{t}\right)}{p_{t}} \frac{\mathrm{d} p_{t}}{c^{2} \mathrm{~d} \rho}$ & $\frac{v_{t}^{2}}{c^{2}}-\frac{v_{r}^{2}}{c^{2}}$ \\
\hline 1 & 0.0 & 0.0880 & 0.0880 & 0.3501 & 0.0187 & 4.3279 & 0.2319 & -0.3313 \\
2 & 0.1 & 0.0872 & 0.0878 & 0.93496 & 0.0187 & 4.3581 & 0.2320 & -0.3309 \\
3 & 0.2 & 0.0848 & 0.0872 & 0.3483 & 0.0186 & 4.4525 & 0.2321 & -0.3296 \\
4 & 0.3 & 0.0808 & 0.0863 & 0.3460 & 0.0184 & 4.6239 & 0.2323 & -0.3275 \\
5 & 0.4 & 0.0752 & 0.0850 & 0.3427 & 0.0182 & 4.8988 & 0.2326 & -0.3245 \\
6 & 0.5 & 0.0678 & 0.0833 & 0.3385 & 0.0179 & 5.3285 & 0.2331 & -0.3206 \\
7 & 0.6 & 0.0586 & 0.0813 & 0.3334 & 0.0175 & 6.0193 & 0.2337 & -0.3158 \\
8 & 0.7 & 0.0474 & 0.0790 & 0.3272 & 0.0171 & 7.2211 & 0.2344 & -0.3101 \\
9 & 0.8 & 0.0342 & 0.0763 & 0.3201 & 0.01767 & 9.6814 & 0.2354 & -0.3034 \\
10 & 0.9 & 0.186 & 0.0732 & 0.3118 & 0.0161 & 17.0758 & 0.2367 & -0.2596 \\
11 & 1.0 & 0.0000 & 0.0689 & 0.3024 & 0.0155 & $\infty$ & 0.2384 & -0.2868 \\
\hline
\end{tabular}

\subsection{Energy Conditions}

Our proposed model of anisotropic fluid satisfies the following energy conditions within the framework of general relativity. 
Table 3. The model parameters for few well known compact stars SAXJ1808.4-3658, HerX-1, 4U1538-52, LMC-X-4, CenX-3, VelaX-1, PSRJ1614-2230 and PSRJ0348+0432.

\begin{tabular}{cccccc}
\hline S.N. & Stellar Objects & $\alpha$ & $\beta$ & $\gamma$ & $\mathscr{T}_{p}$ \\
\hline 1 & SAXJ1808.4-3658 & $1.66 \times 10^{-9}$ & $-0.1979 c$ & $0.9475 c$ & 0.1133 \\
2 & HerX-1 & $1.7825 \times 10^{-9}$ & $-0.215 c$ & $0.9441 c$ & 0.1215 \\
3 & $4 \mathrm{U} 1538-52$ & $2.435 \times 10^{-9}$ & $-0.269 c$ & $0.9344 c$ & 0.1460 \\
4 & LMC-X-4 & $2.552 \times 10^{-9}$ & $-0.3206 c$ & $0.9267 c$ & 0.1674 \\
5 & CenX-3 & $3.112 \times 10^{-9}$ & $-0.4976 c$ & $0.9092 c$ & 0.2287 \\
6 & VelaX-1 & $3.719 \times 10^{-9}$ & $-0.7959 c$ & $0.9034 c$ & 0.2850 \\
7 & PSRJ1614-2230 & $3.6589 \times 10^{-9}$ & $-0.7548 c$ & $0.9028 c$ & 0.2920 \\
8 & PSRJ0348+0432 & $1.213 \times 10^{-9}$ & $-0.4369 c$ & $0.9137 c$ & 0.2096 \\
\hline
\end{tabular}

Null Energy Condition (NEC)

$$
\rho \geq 0
$$

Weak Energy Condition (WEC)

$$
0 \leq \frac{p_{r}}{\rho c^{2}} \leq 1,0 \leq \frac{p_{t}}{\rho c^{2}} \leq 1
$$

Strong Energy Condition (SEC)

$$
0 \leq \frac{p_{r}}{\rho c^{2}}+2 \frac{p_{t}}{\rho c^{2}} \leq 1
$$

The principles of energy condition are plotted graphically against radial distance for realistic stellar objects in Figure 7(a), Figure 7(b) and Figure 7(c).

\subsection{Equilibrium of Different Forces}

There are three kind of forces viz gravitational force $\mathscr{F}_{g}$, hydrostatic force $\mathscr{T}_{h}$ and anisotropical force $\mathscr{F}_{a}$ acting on a compact star which are in equilibrium.

Thus

$$
\mathscr{F}_{g}+\mathscr{F}_{h}+\mathscr{F}_{a}=0
$$

The generalized Tolman-Oppenheimer-Volkoff equaion for equilibrium condition under these forces for anisotropic fluid distribution is given by

$$
-\frac{\mathscr{C l}_{C}(r)\left(\rho c^{2}+p_{r}\right) \mathscr{R}}{r^{2} \mathscr{C}}-\frac{\mathrm{d} p_{r}}{\mathrm{~d} r}+\frac{2\left(p_{t}-p_{r}\right)}{r}=0
$$

where $\mathbb{K}_{\mathrm{g}}(r)$ is effective gravitational mass and is defined by Tolman-Whittaker as

$$
\mathscr{M}_{g}(r)=\frac{r^{2} \cdot \mathscr{A}^{\prime}}{\mathscr{B}}
$$

Now TOV equation reduces to

$$
-\frac{\mathscr{C}^{\prime}\left(\rho c^{2}+p_{r}\right)}{\mathscr{C}}-\frac{\mathrm{d} p_{r}}{\mathrm{~d} r}+\frac{2\left(p_{t}-p_{r}\right)}{r}=0
$$




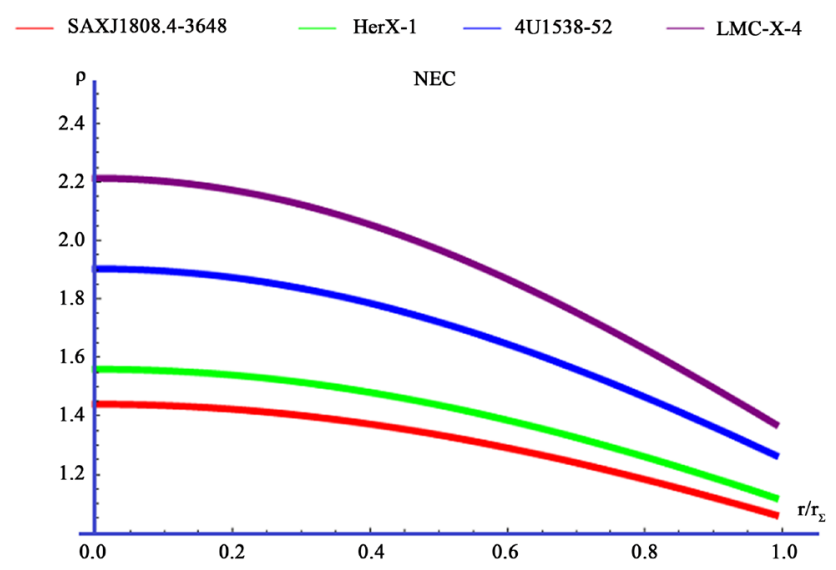

(a)

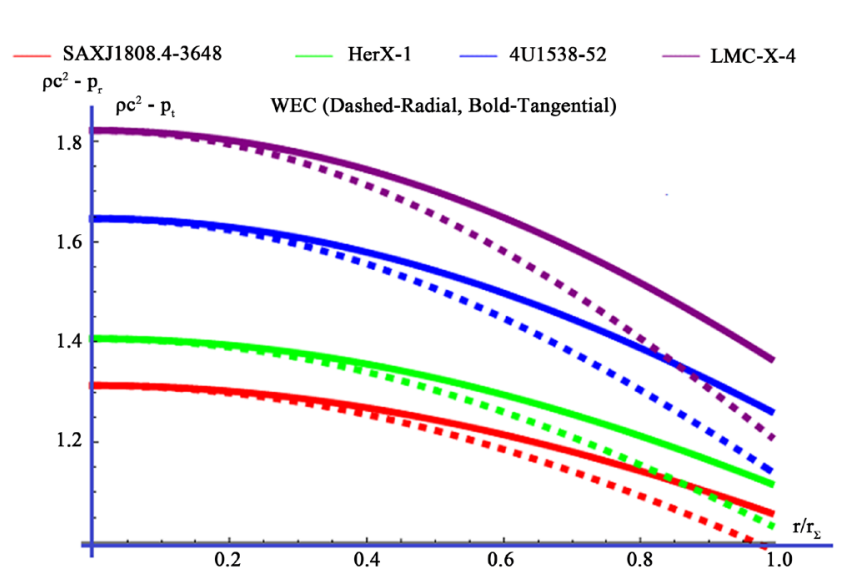

(b)

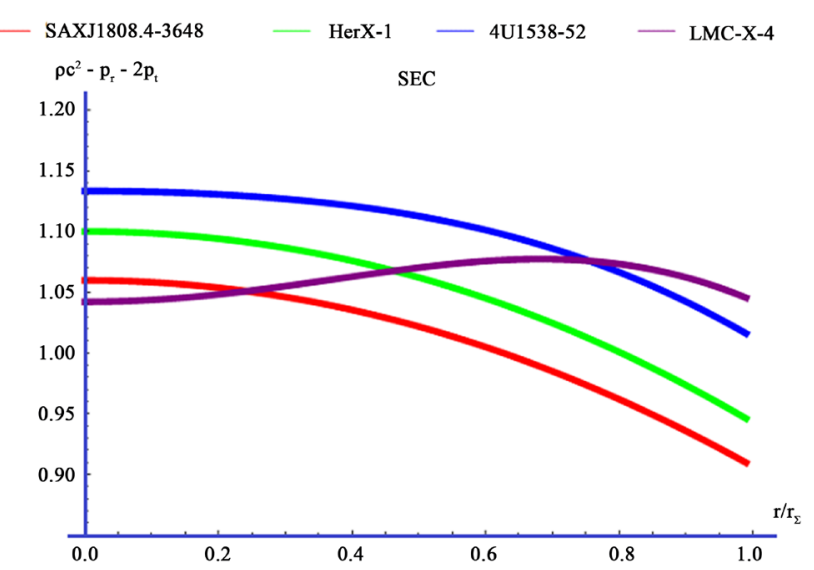

(c)

Figure 7. (a): Variation of NEC with $\frac{r}{r_{\Sigma}} ;(\mathrm{b})$ : Variation of WEC with $\frac{r}{r_{\Sigma}} ;(\mathrm{c})$ : Variation of SEC with $\frac{r}{r_{\Sigma}}$.

In view of Equation (45) and Equation (48) the three forces are given by

$$
\mathscr{F}_{g}=-\frac{\mathscr{C}^{\prime}\left(\rho c^{2}+p_{r}\right)}{\mathscr{d}}
$$

Or

$$
\begin{gathered}
\mathscr{F}_{g}=-\frac{8 \alpha^{2} \gamma r\left(1-\alpha r^{2}\right)\left\{2 \beta\left(1-\alpha r^{2}\right)+3 \gamma\right\}}{\left\{\beta\left(1-\alpha r^{2}\right)+\gamma\right\}} \\
\mathscr{F}_{h}=-\frac{\mathrm{d} p_{r}}{\mathrm{~d} r}
\end{gathered}
$$

Or

$$
\begin{gathered}
\mathscr{F}_{h}=-\alpha^{2} r\left[\frac{8 \gamma\left(1-\alpha r^{2}\right)^{2}\left\{2 \beta\left(1-\alpha r^{2}\right)+3 \gamma\right\}}{\left\{\beta\left(1-\alpha r^{2}\right)+\gamma\right\}^{2}}-12+16 \alpha r^{2}-6 \alpha^{2} r^{4}\right] \\
\mathscr{F}_{a}=\frac{2\left(p_{t}-p_{r}\right)}{r}
\end{gathered}
$$


Or

$$
\mathscr{F}_{a}=\frac{2 \alpha\left(6 \alpha r^{2}-8 \alpha^{2} r^{4}+3 \alpha^{3} r^{6}\right)}{r}
$$

The graphical representations of above mentioned forces and their resultant for astrophysical objects SAXJ1808.4-3658, HerX-1, 4U1538-52 and LMC-X-4 are shown in the Figure 8(a) and Figure 8(b).

\subsection{Causality Conditions}

The causality condition states that the radial and tangential speeds of sound should not be more than one i.e. $0<\frac{v_{r}^{2}}{c^{2}}<1$ and $0<\frac{v_{t}^{2}}{c^{2}}<1$ anywhere within the stellar object. The graphical representations of radial and tangential speeds of sound with respect to $r / r_{\Sigma}$ in the Figure 9(a) and Figure 9(b) which shows that the causality conditions are obeyed throughout the stellar configuration. The condition for potentially stable anisotropic fluid distribution is $-1<\frac{v_{t}^{2}}{c^{2}}-\frac{v_{r}^{2}}{c^{2}}<0$ and $0<\frac{v_{t}^{2}}{c^{2}}-\frac{v_{r}^{2}}{c^{2}}<1$ is the condition for potentially unstable anisotropic fluid distribution. In our relativistic stellar model the stability factor satisfies the condition for potentially stable anisotropic fluid distribution everywhere inside fluid sphere depicted by Figure 10 .

\subsection{Relativistic Adiabatic Index}

The relativistic adiabatic index for anisotropic fluid distribution of an astrophysical object is given by

$$
\Gamma_{r}=\frac{\rho c^{2}+p_{r}}{p_{r}} \frac{\mathrm{d} p_{r}}{c^{2} \mathrm{~d} \rho} \text { and } \Gamma_{t}=\frac{\rho c^{2}+p_{t}}{p_{t}} \frac{d p_{t}}{c^{2} \mathrm{~d} \rho}
$$

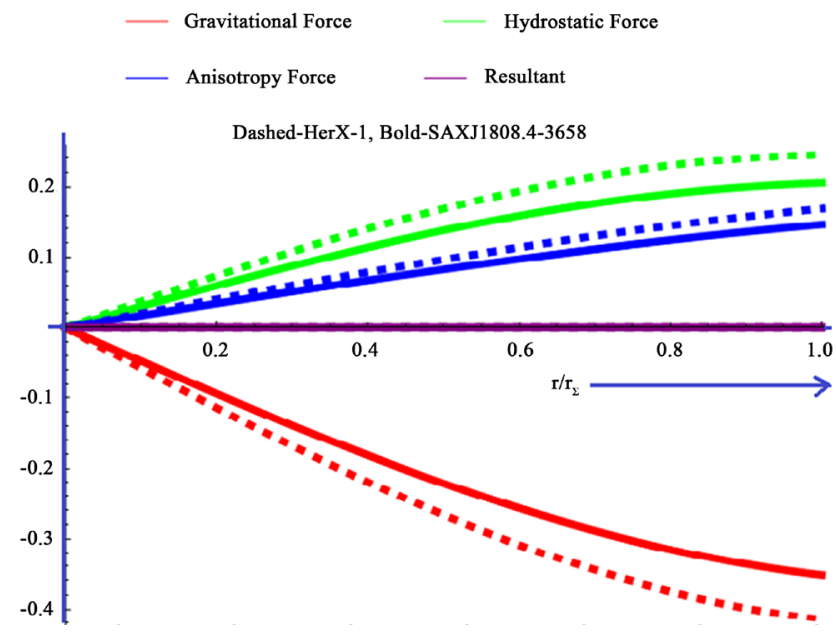

(a)

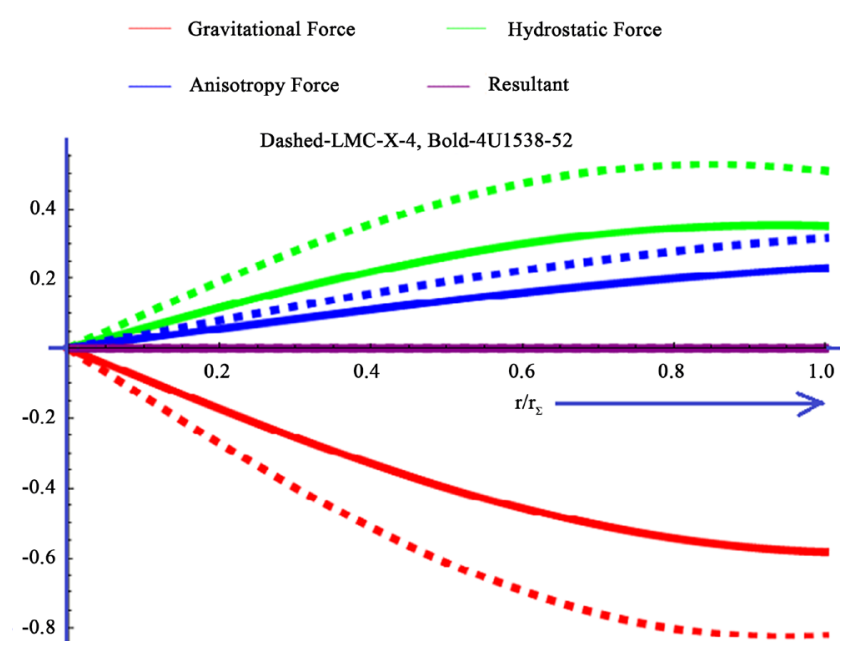

(b)

Figure 8. (a) Variation of three forces $\mathscr{F}_{g}, \mathscr{F}_{h}$ and $\mathscr{F}_{a}$ with $\frac{r}{r_{\Sigma}}$ (b): Variation of three forces $\mathscr{F}_{g}, \mathscr{F}_{h}$ and $\mathscr{F}_{a}$ with $\frac{r}{r_{\Sigma}}$. 


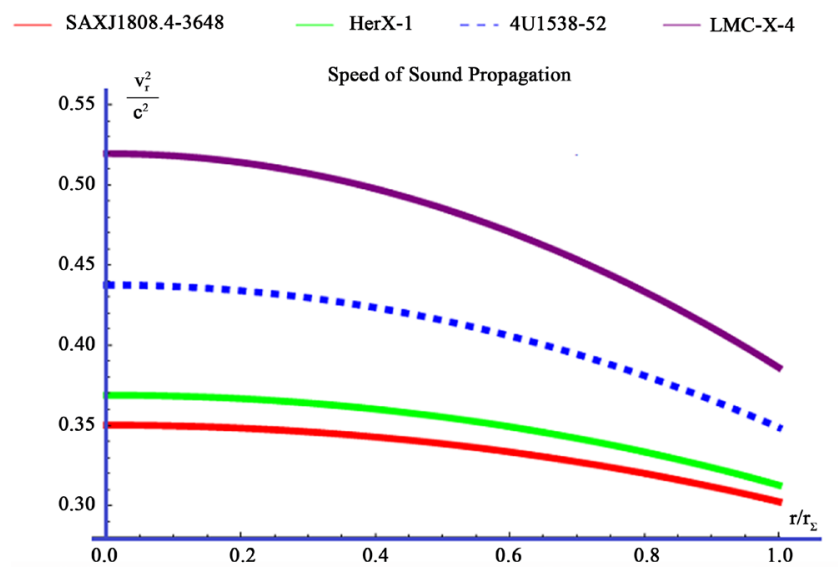

(a)

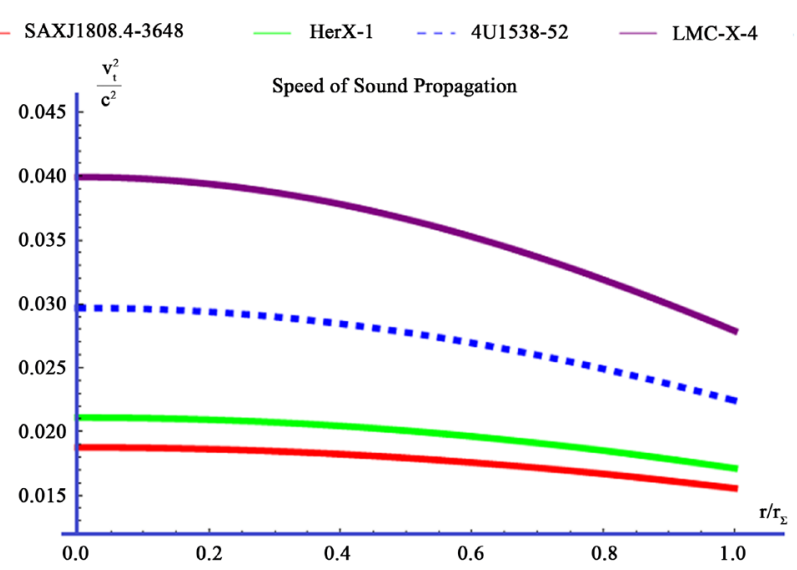

(b)

Figure 9. (a): Variation of $\frac{\mathrm{d} p_{r}}{c^{2} \mathrm{~d} \rho}$ with $\frac{r}{r_{\Sigma}}$; (b): Variation of $\frac{\mathrm{d} p_{t}}{c^{2} \mathrm{~d} \rho}$ with $\frac{r}{r_{\Sigma}}$.

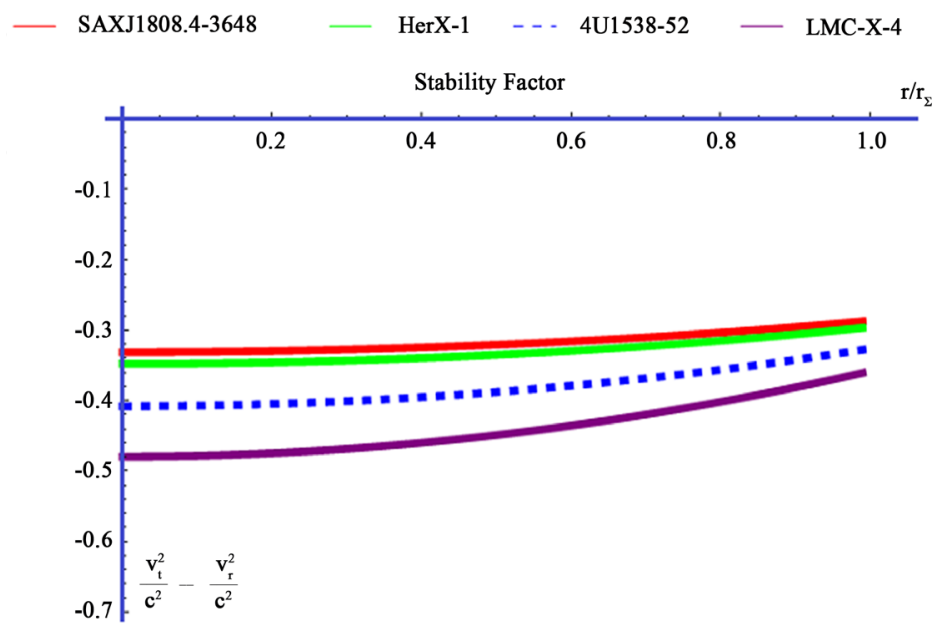

Figure 10. Variation of Stability factor $\left(\frac{v_{t}^{2}}{c^{2}}-\frac{v_{r}^{2}}{c^{2}}\right)$ with $\frac{r}{r_{\Sigma}}$.

In our model the variation of radial and tangential adiabatic indices with $\frac{r}{r_{\Sigma}}$ are represented graphically in Figure 11(a) and Figure 11(b). The radial relativistic adiabatic index $\Gamma_{r}$ is greater than $\frac{4}{3}$.

\subsection{Harrison Zeldovich Novikov Static Stability Criterion}

Harrison [45] Zeldovich Novikov [46] static stability criterion are given by

$$
\begin{aligned}
& \frac{\mathrm{d} / /}{\mathrm{d} \rho_{0}}>0 \text { for stable fluid distribution, } \\
& \frac{\mathrm{d} / /}{\mathrm{d} \rho_{0}}<0 \text { for unstable fluid distribution, }
\end{aligned}
$$

In our model the mass of configuration in terms of central density is given by 


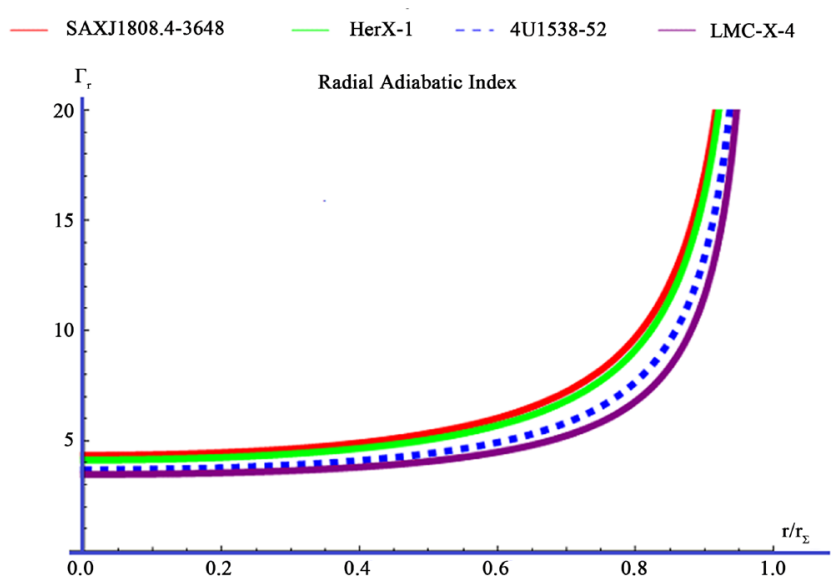

(a)

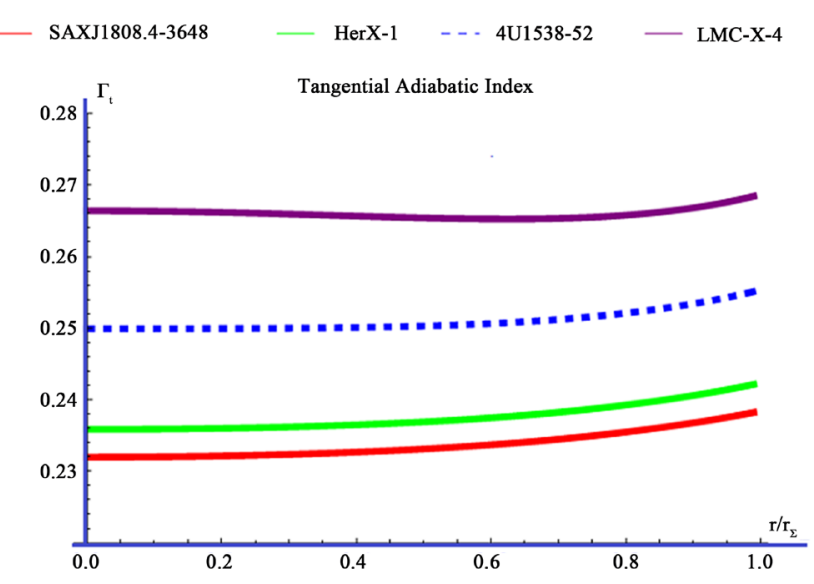

(b)

Figure 11. (a): Variation of $\frac{\left(\rho c^{2}+p_{r}\right)}{p_{r}} \frac{\mathrm{d} p_{r}}{c^{2} \mathrm{~d} \rho}$ with $\frac{r}{r_{\Sigma}}$; (b): Variation of $\frac{\left(\rho c^{2}+p_{t}\right)}{p_{r}} \frac{\mathrm{d} p_{t}}{c^{2} \mathrm{~d} \rho}$ with $\frac{r}{r_{\Sigma}}$.

$$
\left(1-\frac{2}{c^{2} r_{\Sigma}}\right)^{\frac{3}{2}}\left[-4+5\left(1-\frac{2}{c^{2} r_{\Sigma}}\right)^{\frac{1}{2}}\right]=\frac{8 \pi}{c^{2}} \rho_{0} r_{\Sigma}^{2}-1
$$

The rate of change of mass of stellar configuration with respect to central density is always positive. Consequently our solution gives stable stellar configuration. The variation of masses of astrophysical objects SAXJ1808.4-3658, HerX-1, 4U1538-52 and LMC-X-4 with their central density is graphically represented in Figure 12.

\section{Conclusions}

We have given a well behaved analytic charge free solution for spherical and symmetric anisotropic fluid distribution given by Sah and Chandra [28] in addition with energy conditions, variation of mass with central density, equation of state and mass-radius relationship in contest of some astrophysical objects, SAXJ1808.4-3658, HerX-1, 4U1538-52, LMC X-4, CenX-3, VelaX-1, PSRJ1614-2230 and PSRJ0348+0432. Throughout the paper all the calculations, Table 1 and Table 2 are made for astrophysical object SAXJ1808.4-3658. The graphs are drawn for the compact star SAXJ1808.4-3658, HerX-1, 4U1538-52 and LMC X-4. It is concluded that the physical properties pressures, mass energy density and redshift with positive central value decrease monotonically with increasing radial distance and the causality condition is satisfied throughout within super dense fluid distribution.

By using suitable model parameters given in Table 3, we have calculated the mass and radius of above mentioned compact stars presented in Table 4 and it is clear that calculated values of mass and radius of these stars is well fitted with the observational data. We have also obtained the central density, surface density, central pressure, and surface redshift given in Table 5. The compactness factor is 


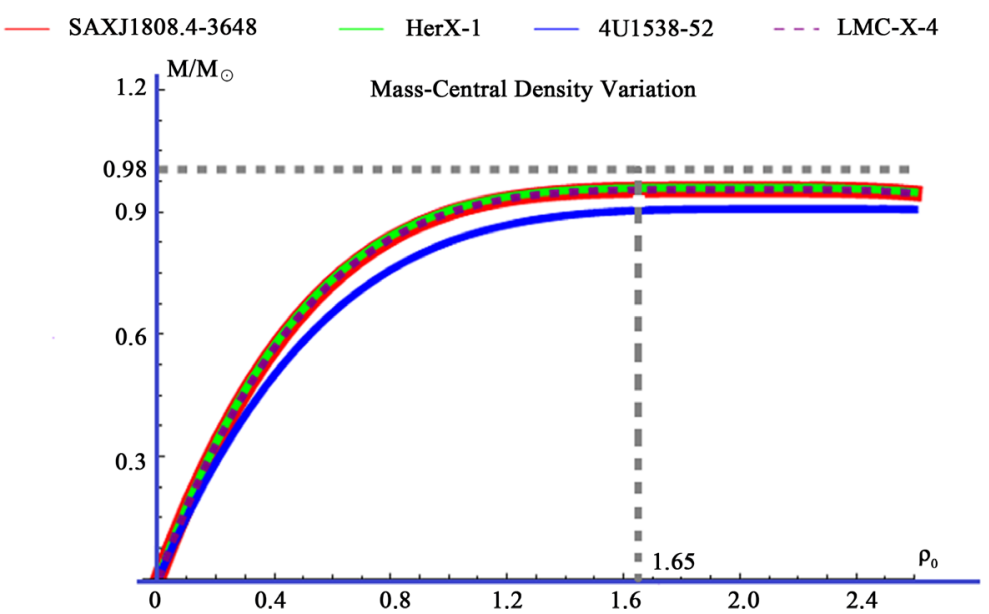

Figure 12. Variation of mass with central density.

Table 4. The Observed and calculated values of mass and radius of few compact stars SAXJ1808.4-3658, HerX-1, 4U1538-52, LMC-X-4, CenX-3, VelaX-1, PSRJ1614-2230 and PSRJ0348+0432.

\begin{tabular}{|c|c|c|c|c|c|c|}
\hline S.N. & Stellar Objects & $.11 .1 / 0$ & $r_{\Sigma}($ in $\mathrm{km})$ & $.11 / .1 / 0$ & $r_{\Sigma}($ in $\mathrm{km})$ & Referance \\
\hline & & (Observed) & (Observed) & (Calculated) & (Calculated) & \\
\hline 1 & SAXJ1808.4-3658 & $0.9 \pm 0.3$ & $7.951 \pm 1.0$ & 0.65 & 8.50 & Elebert et al. [47] \\
\hline 2 & HerX-1 & $0.85 \pm 0.15$ & $8.1 \pm 0.45$ & 0.70 & 8.54 & $\begin{array}{c}\text { Abubekerov et al. } \\
{[48]}\end{array}$ \\
\hline 3 & $4 \mathrm{U} 1538-52$ & $0.87 \pm 0.07$ & $7.866 \pm 0.21$ & 0.80 & 8.07 & Rawls et al. [49] \\
\hline 4 & LMC-X-4 & $1.04 \pm 0.09$ & $8.301 \pm 0.2$ & 0.96 & 8.50 & Rawls et al. [49] \\
\hline 5 & CenX-3 & $1.49 \pm 0.08$ & $9.178 \pm 0.13$ & 1.42 & 9.20 & Rawls et al. [49] \\
\hline 6 & VelaX-1 & $1.77 \pm 0.08$ & $9.56 \pm 0.08$ & 1.85 & 9.62 & Rawls et al. [49] \\
\hline 7 & PSRJ1614-2230 & $1.97 \pm 0.04$ & $9.69 \pm 0.2$ & 1.94 & 9.85 & $\begin{array}{c}\text { Demorest et al. } \\
{[50]}\end{array}$ \\
\hline 8 & PSRJ0348+0432 & $2.01 \pm 0.04$ & $13.0 \pm 2.0$ & 1.98 & 14.00 & $\begin{array}{c}\text { Demorest et al. } \\
{[50]}\end{array}$ \\
\hline
\end{tabular}

Table 5. The Central Density, Surface Density, Central Pressure and Surface Red Shift of compact stars SAXJ1808.4-3658, HerX-1, 4U1538-52, LMC-X-4, CenX-3, VelaX-1, PSRJ1614-2230 and PSRJ0348+0432.

\begin{tabular}{cccccc}
\hline S.N. & StellarObjects & $\begin{array}{c}\text { Central Density } \\
\left(\text { in gm } / \mathrm{cm}^{3}\right)\end{array}$ & $\begin{array}{c}\text { Surface Density } \\
\left(\mathrm{in} \mathrm{gm} / \mathrm{cm}^{3}\right)\end{array}$ & $\begin{array}{c}\text { Central Pressure } \\
\left.\text { (in dyne } / \mathrm{cm}^{2}\right)\end{array}$ & Red Shift \\
\hline 1 & SAXJ1808.4-3658 & $1.07 \times 10^{15}$ & $0.784 \times 10^{15}$ & $0.847 \times 10^{35}$ & 0.138 \\
2 & HerX-1 & $1.15 \times 10^{15}$ & $0.819 \times 10^{15}$ & $1.027 \times 10^{35}$ & 0.149 \\
3 & $4 \mathrm{U} 1538-52$ & $1.57 \times 10^{15}$ & $1.034 \times 10^{15}$ & $1.093 \times 10^{35}$ & 0.188 \\
4 & LMC-X-4 & $1.645 \times 10^{15}$ & $1.009 \times 10^{15}$ & $1.582 \times 10^{35}$ & 0.226 \\
5 & CenX-3 & $2.006 \times 10^{15}$ & $0.982 \times 10^{15}$ & $6.938 \times 10^{35}$ & 0.357 \\
6 & VelaXv1 & $2.431 \times 10^{15}$ & $0.923 \times 10^{15}$ & $3.956 \times 10^{35}$ & 0.525 \\
7 & PSRJ1614-2230 & $2.358 \times 10^{15}$ & $0.879 \times 10^{15}$ & $3.608 \times 10^{35}$ & 0.551 \\
8 & PSRJ0348+0432 & $0.781 \times 10^{15}$ & $0.412 \times 10^{15}$ & $2.149 \times 10^{35}$ & 0.312 \\
\hline
\end{tabular}




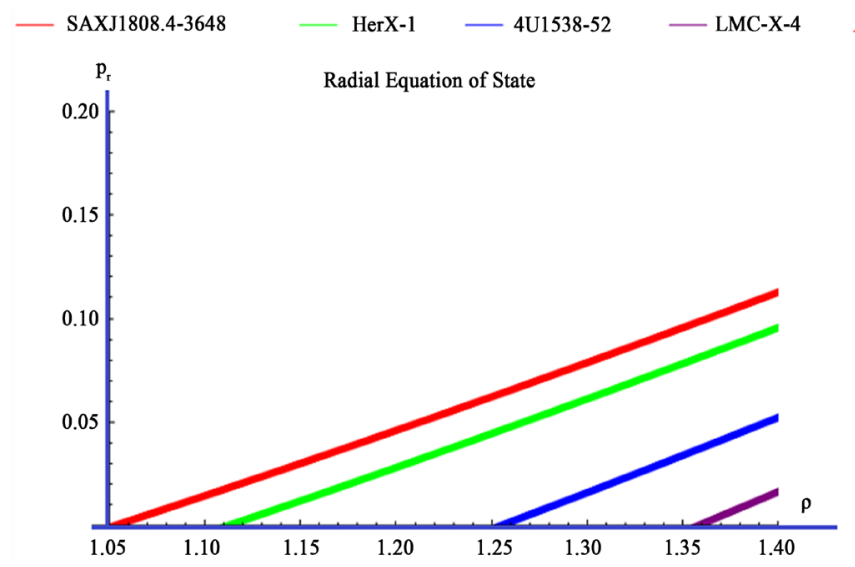

(a)

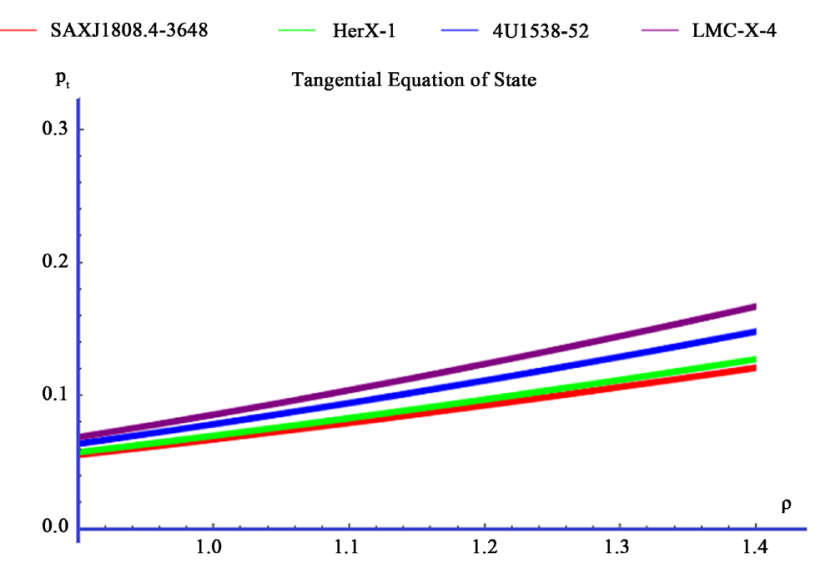

(b)

Figure 13. (a): Variation of radial pressure $p_{r}$ with density $\rho$ against $r$, (b): Variation of tangential pressure $p_{t}$ with density $\rho$ against $r$.

always less than $\frac{4}{9}$. The red shift is also satisfied the upper bound limit for the realistic anisotropic star models i.e. $z_{s} \leq 1$. For the EoS, we plot a graph for $p_{r}$ and $p_{t}$ versus against $r$ as shown in Figure 13(a) and Figure 13(b).

It is found that the present model is very close to the observed data of a number of compact stars like SAXJ1808.4-3658, HerX-1, 4U1538-52, LMC X-4, CenX-3, VelaX-1, PSRJ1614-2230 and PSRJ0348+0432 and many more given by Elebert et al. [47], Abubekerov et. al. [48], Rawls et al. [49], Demorest et al. [50] and Gangopadhyay et al. [51]. Thus the solution obtained by us is very helpful in constructing the models of super dense astrophysical object like Neutron stars, Strange star, quark stars and many more.

\section{References}

[1] Schwarzschild, K. (1916) On the Gravitational Field of a Mass Point According to Einstein's Theory. Sitzer. Preuss. Akad. Wiss., Berlin, 189-196.

[2] Tolman, R.C. (1939) Static Solutions of Einstein's Field Equations for Spheres of Fluid. Physical Review, 55, 364-373. https://doi.org/10.1103/PhysRev.55.364

[3] Buchdahl, H.A. (1959) General Relativistic Fluid Spheres. Physical Review D, 116, 1027-1034. https://doi.org/10.1103/PhysRev.116.1027

[4] Delgaty, M.S.R. and Lake, K. (1998) Physical Acceptability of Isolated, Static, Spherically Symmetric, Perfect Fluid Solutions of Einstein's Equations. Computer Physics Communications, 115, 395-415. https://doi.org/10.1016/S0010-4655(98)00130-1

[5] Patwardhan, G.K. and Vaidya, P.C. (1943) Relativistic Distributions of Matter of Radial Symmetry. Journal of the University of Bombay, 12, 23.

[6] Mehra, A.L. (1966) Radially Symmetric Distribution of Matter. Journal of the Australian Mathematical Society, 6, 153-156.

https://doi.org/10.1017/S1446788700004730

[7] Kuchowicz, B. (1968) General Relativistic Fluid Spheres. II. Solutions of the Equation for e- $\lambda$. Acta Physica Polonica, 34, 131. 
[8] Matese, J.J. and Whitman, P.G. (1980) New Method for Extracting Static Equilibrium Configurations in General Relativity. Physical Review D, 22, 1270-1275. https://doi.org/10.1103/PhysRevD.22.1270

[9] Durgapal, M.C. (1982) A Class of New Exact Solutions in General Relativity. Journal of Physics A: Mathematical and General, 15, 2637-1644. https://doi.org/10.1088/0305-4470/15/8/039

[10] Nariai, S. (1950) On Some Static Solutions of Einstein's Gravitational Field Equations in a Spherically Symmetric Case. The Science Reports of the Tohoku University, 134, 160 .

[11] Goldman, S.P. (1978) Physical Solutions to General-Relativistic Fluid Sphere. The Astrophysical Journal, 226, 1079-1086. https://doi.org/10.1086/156684

[12] Adler, R.J. (1974) A Fluid Sphere in General Relativity. Journal of Mathematical Physics, 15, 727-729. https://doi.org/10.1063/1.1666717

[13] Heintzmann, H.Z. (1969) Newtonian Time in General Relativity. Zeitschrift für Physik, 228, 489-493. https://doi.org/10.1007/BF01558346

[14] Finch, N.R. and Skea, J.E.F. (1989) A Realistic Stellar Model Based on an Ansatz of Duorah and Ray. Class Quantum Gravity, 6, 467-476. https://doi.org/10.1088/0264-9381/6/4/007

[15] Maurya, S.K. and Gupta, Y.K. (2013) Charged Fluid to Anisotropic Fluid Distribution in General Relativity. Astrophysics and Space Science, 344, 243-251. https://doi.org/10.1007/s10509-012-1302-4

[16] Pant, N. (2011) Some New Exact Solutions with Finite Central Parameters and Uniform Radial Motion of Sound. Astrophysics and Space Science, 331, 633-644. https://doi.org/10.1007/s10509-010-0453-4

[17] Ivanov, B.V. (2012) Collapsing Shear-Free Perfect Fluid Spheres with Heat Flow. General Relativity and Gravitation, 44, 1835-1855. https://doi.org/10.1007/s10714-012-1370-3

[18] Ivanov, B.V. (2002) Static Charged Perfect Fluid Spheres in General Relativity. Physical Review D, 65, Article ID: 104001. https://doi.org/10.1103/PhysRevD.65.104001

[19] Pant, N., Fuloria, P. and Tewari, B.C. (2012) A New Well Behaved Exact Solution in General Relativity for Perfect Fluid. Astrophysics and Space Science, 340, 407-412. https://doi.org/10.1007/s10509-012-1068-8

[20] Sah, A. and Chandra, P. (2016) Spherical Anisotropic Fluid Distribution in General Relativity. World Journal of Mechanics, 6, 487-504. http://dx.doi.org/10.4236/wjm.2016.612034

[21] Komathiraj, K. and Maharaj, S.D. (2007) Tikekar Superdense Stars in Electric Fields. Journal of Mathematical Physics, 48, Article ID: 042501. https://doi.org/10.1063/1.2716204

[22] Herrera, L., Ospino, J. and Di Perisco, A. (2008) All Static Spherically Symmetric Anisotropic Solutions of Einstein's Equations. Physical Review D, 77, Article ID: 027502. https://doi.org/10.1103/PhysRevD.77.027502

[23] Thirukkanesh, S. and Ragel, F.C. (2012) Exact Anisotropic Sphere with Polytropic Equation of State. Pramana-Journal of Physics, 78, 687-696.

[24] Chaisi, M. and Maharaj, S.D. (2006) Anisotropic Static Solutions in Modelling Highly Compact Bodies. Pramana-Journal of Physics, 66, 609-614.

https://doi.org/10.1007/BF02704504 
[25] Sunzu, J.M., Maharaj, S.D. and Ray, S. (2014) Charged Anisotropic Models for Quark Stars. Astrophysics Space Science, 352, 719-727. https://doi.org/10.1007/s10509-014-1918-7

[26] Maurya, S.K. and Gupta, Y.K. (2012) A Family of Anisotropic Super-Dense Star Models using a Space-Time Describing Charged Perfect Fluid Distributions. Physica Scripta, 86, Article ID: 025009. https://doi.org/10.1088/0031-8949/86/02/025009

[27] Bhar, P., Singh, K.N. and Manna, T. (2017) A New Class of Relativistic Model of Compact Stars of Embedding Class I. International Journal of Modern Physics D, 26, Article ID: 1750090. https://doi.org/10.1142/S0218271817500900

[28] Sah, A. and Chandra, P. (2016) Class of Charged Fluid Balls in General Relativity. International Journal of Astronomy and Astrophysics, 6, 494-511. https://doi.org/10.4236/ijaa.2016.64038

[29] Whitman, P.G. and Burch, R.C. (1981) Charged Spheres in General Relativity. Physical Review D, 24, 2049. https://doi.org/10.1103/PhysRevD.24.2049

[30] Tikekar, R. (1984) Spherical Charged Fluid Distributions in General Relativity. Journal of Mathematical Physics, 25, 1481-1483. https://doi.org/10.1063/1.526318

[31] Ivanov, B.V. (2017) Analytical Study of Anisotropic Compact Star Models. The European Physical Journal C, 77, 738. https://doi.org/10.1140/epjc/s10052-017-5322-7

[32] Gupta, Y.K. and Kumar, M. (2005) A Superdense Star Model as Charged Analogue of Schwarzschild's Interior Solution. General Relativity and Gravitation, 37, 575-583. https://doi.org/10.1007/s10714-005-0043-x

[33] Herrera, L., Ospino, J., Di Parisco, A., Fuenmayor, E. and Triconis, O. (2009) Structure and Evolution of Self-Gravitating Objects and the Orthogonal Splitting of the Riemann Tensor. Physical Review D, 79, Article ID: 064025.

https://doi.org/10.1103/PhysRevD.79.064025

[34] Herrera, L., Di Parisco, A., Hern'ndez-Pastora, J.L. and Santos, N.O. (1998) On the Role of Density Inhomogeneity and Local Anisotropy in the Fate of Spherical Collapse. Physics Letters A, 237, 113-118. https://doi.org/10.1016/S0375-9601(97)00874-8

[35] Herrera, L. and Santos, N.O. (1997) Local Anisotropy in Self-Gravitating Systems. Physics Reports, 286, 53-130. https://doi.org/10.1016/S0370-1573(96)00042-7

[36] Tewari, B.C. and Charan, K. (2015) Gravitational Collapse, Shear-Free Anisotropic Radiating Star. Astrophysics and Space Science, 357, Article ID: 107. https://doi.org/10.1007/s10509-015-2335-2

[37] Tewari, B.C. and Charan, K. (2015) Dissipative Spherical Gravitational Collapse of Isotropic Fluid. Journal of Modern Physics, 6, Article No. 55006.

[38] Tewari, B.C., Charan, K. and Rani, J. (2016) Spherical Gravitational Collapse of Anisotropic Radiating Fluid Sphere. International Journal of Astronomy and Astrophysics, 6, 155-165.

[39] Tewari, B.C. (2013) Collapsing Shear-Free Radiating Fluid Spheres. General Relativity and Gravitation, 45, 1547-1558. https://doi.org/10.1007/s10714-013-1545-6

[40] Ivanov, B.V. (2010) Evolving Spheres of Shear-Free Anisotropic Fluid. International Journal of Modern Physics A, 25, 3975-3991. https://doi.org/10.1142/S0217751X10050202

[41] Ivanov, B.V. (2011) Self-Gravitating Spheres of Anisotropic Fluid in Geodesic Flow. International Journal of Modern Physics D, 20, Article No. 319. https://doi.org/10.1142/S0218271811018858 
[42] Ivanov, B.V. (2016) A Different Approach to Anisotropic Spherical Collapse with Shear and Heat Radiation. International Journal of Modern Physics D, 25, Article ID: 1650049. https://doi.org/10.1142/S0218271816500498

[43] Ivanov, B.V. (2016) All Solutions for Geodesic Anisotropic Spherical Collapse with Shear and Heat Radiation. Astrophysics and Space Science, 361, Article ID: 18. https://doi.org/10.1007/s10509-015-2603-1

[44] Maurya, S.K. and Gupta, Y.K. (2014) A New Class of Relativistic Charged Anisotropic Super Dense Star Models. Astrophysics and Space Science, 353, 657-665. https://doi.org/10.1007/s10509-014-2041-5

[45] Harrison, B.K., Thorne, K.S. and Wakano, M. (1965) Gravitational Theory and Gravitational Collapse. University of Chicago Press, Chicago.

[46] Zeldovich, Y.B. and Novikov, I.D. (1971) Relativistic Astrophysics Vol. 1: Stars and Relativity. University of Chicago Press, Chicago.

[47] Elebert, P., Reynolds, M.T., Callanan, P.J., Hurley, D.J., Ramsay, G., Lewis, F., Russell, D.M., Nord, B., Kane, S.R., DePoy, D.L. and Hakala, P. (2009) Optical Spectroscopy and Photometry of SAX J1808.4-3658 in Outburst. Monthly Notices of the Royal Astronomical Society, 395, 884-894. https://doi.org/10.1111/j.1365-2966.2009.14562.x

[48] Abubekerov, M.K., Antokhina, E.A., Cherepashchuk, A.M. and Shimanskii, V.V. (2008) The Mass of the Compact Object in the X-Ray Binary Her X-1/HZ Her. Astronomy Reports, 52, 379-389. https://doi.org/10.1134/S1063772908050041

[49] Rawls, M.L., Orosz, J.A., McClintock, J.E., Torres, M.A.P., Bailyn, C.D. and Buxton, M.M. (2011) Refined Neutron Star Mass Determinations for Six Eclipsing X-Ray Pulsar Binaries. The Astrophysical Journal, 730, Article ID: 25. https://doi.org/10.1088/0004-637X/730/1/25

[50] Demorest, P., Pennucci, T., Ransom, S., Roberts, M. and Hessels, J. (2010) Shapiro Delay Measurement of a Two Solar Mass Neutron Star. Nature, 467, 1081-1083. https://doi.org/10.1038/nature09466

[51] Gangopadhyay, T., Ray, S., Li, X.D., Dey, J. and Dey, M. (2013) Strange Star Equation of State Fits the Refined Mass Measurement of 12 Pulsars and Predicts Their Radii. Monthly Notices of the Royal Astronomical Society, 431, 3216-3221. https://doi.org/10.1093/mnras/stt401 